\title{
COX-2 inhibitors and the gastrointestinal tract
}

\author{
I Bjarnason, K D Rainsford*
}

The discovery of cyclooxygenase 2 (COX-2) a decade ago heralded one of the most rapid and expensive development and marketing of a new class of drug that we have witnessed. This has resulted in publication of an interesting mixture of exceptionally high quality basic and clinical research. Based on the COX dogma, that COX-1 is good and COX-2 is bad, we were promised equal therapeutic efficacy to conventional nonsteroidal inflammatory drugs (NSAIDs) with the COX-2 selective inhibitors and absence of gastrointestinal side effects that otherwise represent a significant public health problem. Have these promises come to fruition?

The semantic problem about the term COX-2 selective agents is acknowledged, ${ }^{1-3}$ but for our purpose (accepting that nimesulide and etodolac, at least, have a case for using this label) our discussion relates to celecoxib and rofecoxib as data on these agents are now abundant.

Gastroenterologists are usually unconcerned about therapeutic efficacy of anti-inflammatory analgesics but the COX-2 selective agents have equivalent efficacy to conventional NSAIDs and no new unexpected side effects have been encountered. Curiously, the prevalence of dyspeptic symptoms are similar to conventional NSAIDs. ${ }^{4}$ The COX-2 selective agents have otherwise come through the conventional gastroduodenal safety assessments with flying colours.

- Equivalent short term endoscopy damage to placebo in volunteers, even at very high doses. ${ }^{5}$

- Equivalent long term endoscopy damage to placebo in patients. ${ }^{6}$

- Significant reduction (about $60 \%$ ) in serious outcomes (perforation, bleeds) in patients taking the drugs long term (reported at DDW, San Diego).

Also, nimesulide and rofecoxib (in press) cause no short term small bowel damage in healthy volunteers which is a good predictor of long term tolerability. Is this the proof to the COX dogma or are there still some concerns?

Of special note is the high (3-11\%) prevalence of gastric damage in the placebo arms of the long term endoscopy studies. Some of this damage may be due to concomitant ingestion of aspirin, used for cardiovascular prophylaxis.

Interestingly, the normal intestinal appearances in COX-1 knockout animals rang warning bells for the COX dogma for some of us. It is therefore particularly

Department of Medicine, Guy's, King's, St Thomas' Medical School, London, UK and *Department of Biological Sciences, Sheffield Hallam University, Sheffield, UK

Correspondence to: Dr I Bjarnason, Department of Medicine, Guy's, King's, St Thomas's Medical School, Bessemer Road, London SE5 9PJ, UK. ingvar.bjarnason@kcl.ac.uk interesting that in the absence of a "topical" effect (Peter Isakson, personal communication, 1999 and widely confirmed at the DDW 2000), selective COX-1 inhibition (SC-560) is not associated with gastrointestinal damage. Rather it is the dual inhibition of COX-1 and COX-2 that is important. These deviations from the COX dogma pose potential problems as even mini doses of aspirin inhibit gastric COX-1 almost completely. The precise importance of concomitant aspirin ingestion and COX-2 inhibitory agents demands further study but these findings should also be a stimulus to develop selective COX-1 inhibitors that may be devoid of the gastric toxicity of aspirin.

Selective COX-2 inhibition does not therefore appear to cause significant new gastrointestinal damage in humans. Their possible detrimental effect on pre-existing intestinal disease, ${ }^{7}$ however, requires further clarification. COX-2 selective agents delay healing of experimental gastric ulcers in animals and if substantiated in humans may have implications for patients with Helicobacter pylori driven gastroduodenal ulcer diathesis. Secondly, COX-2 inhibitors may exacerbate $^{8}$ or ameliorate ${ }^{9}$ the severity of experimental colitis in rodents. This needs to be studied in humans as NSAIDs may cause relapse of inflammatory bowel disease and many of these patients require antiinflammatory analgesics for arthritis, metabolic bone disease, etc. NSAIDs play a detrimental role in some other intestinal diseases and it is also possible that selective COX-2 inhibitors may perpetuate NSAID induced damage. This should not, however, deter from the fact that on current evidence it is likely that selective COX-2 inhibitors will transform the care of arthritic patients. For many this development has not come too soon.

1 Jacob M, Simpson R, Bjarnason I. Nonsteroidal anti-inflammatory drugs, cyclooxygenase selectivity and gastrointestinal toxicity. Ital $\mathcal{f}$ Gastroenterol Hepatol 1998;30:12-18.

2 Scarpignatio C, Bjarnason I, Bretagne J-F, et al. Towards a GI safer antiinflammatory therapy. Gastroenterol Int 1999;12:180-215.

3 Brooks P, Emery P, Evans JF, et al. Interpreting the clinical significance of the differential inhibition of cyclooxygenase- 1 and cyclooxygenase2. Rheumatology 1999;38:779-88.

4 Cannon GW, Caldwell JR, Holt P, et al. Rofecoxib, a specific inhibitor of cyclooxygenase 2, with clinical efficacy comparable with that of diclofenac sodium: results of a one-year, randomized, clinical trial in diclofenac sodium: results of a one-year, randomized, clinical trial in patients with osteoarthritis of the knee and hip. Rofecoxib Pha
Protocol 035 Study Group. Arthritis Rheum 2000;43:978-87.

5 Lanza FL, Rack MF, Simon TJ, et al. Specific inhibition of cyclooxygenase-2 with MK-0966 is associated with less gastroduodenal damage than either aspirin or ibuprofen. Aliment Pharmacol The 1999;13:761-7.

6 Simon LS, Weaver AL, Graham DY, et al. Anti-inflammatory and upper gastrointestinal effects of celecoxib in rheumatoid arthritis: a randomised controlled trial. $7 A M A$ 1999;282:1921-8.

7 Bjarnason I, Hayllar J, Macpherson AJ, et al. Side effects of nonsteroidal anti-inflammatory drugs on the small and large intestine. Gastroenterology 1993;104:1832-47.

8 Reuter BK, Asfaha S, Buret A, et al. Exacerbation of inflammationassociated colonic injury in rat through inhibition of cyclooxygenase-

9 Karmeli F, Cohen P, Rachmilewitz D. Cyclo-oxygenase-2 inhibitors ameliorate the severity of experimental colitis in the rat. Eur $\mathcal{F}$ Gastroenterol Hepatol 1999;12:223-31. 